\title{
The Effects of Short-Term Resistance Training with \& without Blood Flow Restriction on Neuromuscular Adaptations
}

Gabriela A. Soto, Roberto Osornio IV, Danny Dominguez, Delisha Horne, Ashley Taylor, Pedro Vasquez, Hugo Santana, Murat Karabulut.

Laboratory of Exercise Physiology; College of Health Affairs; University of Texas Rio Grande Valley; Brownsville, TX

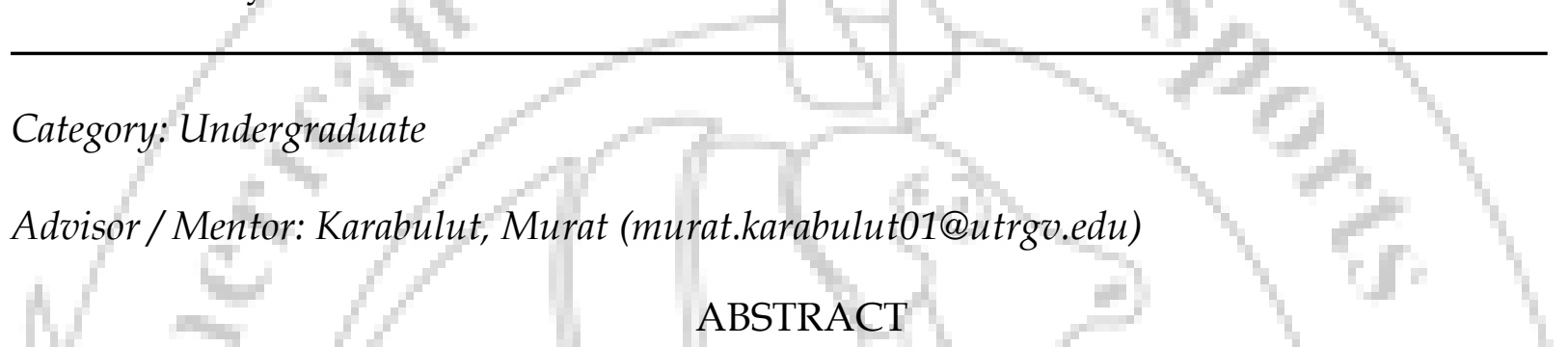

Initial changes in muscle strength following resistance training are proven to be due to neuromuscular adaptations in muscle fibers. For Health Fitness Specialists, there will always be a need to discover new methods for improving neuromuscular adaptations in potential clients. Resistance training with blood

flow restriction is a relatively new method of training that promotes neuromuscular adaptation.

PURPOSE: To investigate the neuromuscular adaptations in quadriceps muscles after short-term resistance training with and without blood flow restriction (BFR). METHODS: Twelve males (age $=27.4 \pm$ 6.3 years; height $=171 \pm 7 \mathrm{~cm}$; weight $=79.8 \pm 13.2 \mathrm{~kg}$ ) volunteered to participate in this study. Subjects had their legs randomly assigned to two training conditions that differed in contraction intensity. One leg was trained with blood flow restriction (BFR) at an intensity of $20 \%$ of their one repetition maximum (1RM) for a total of four sets $(30,15,15,15$ repetitions) and the contralateral leg was trained without BFR (non-BFR) at an intensity of 70\% 1RM for two sets of 11 repetitions. Subjects performed unilateral knee extensions and trained each leg with their assigned training protocol for 2 weeks, 3 times/wk. Pre, and post $1 \mathrm{RM}$ tests were performed for each leg on a dynamic constant external resistance machine and an isokinetic exercise machine was used to determine maximal voluntary contraction (MVC) and isokinetic exercises at two speeds of $60^{\circ} / \mathrm{s}$ and $180^{\circ} / \mathrm{s}$. Additionally, heart rate (HR) and rating of perceived exertion

(RPE) were recorded after the completion of each set. RESULTS: No condition*time interaction or condition main effect for 1RM strength test was detected, but there was a significant time main effect for $1 R M$ strength from pre to post values $(\mathrm{p}=0.01)$. There were significant condition*time, condition*day, and day*time interactions and condition (HR was higher for non-BFR and RPE for BFR), day, and time main effects for HR and RPE values (set 3 and 4 for BFR vs. set 1 and 2 for non-BFR) $(p<0.05)$. There were no significant condition*time interaction, condition main effect, or time main effect for MVC and isokinetic strength at $180^{\circ} / \mathrm{s}$ and $60^{\circ} / \mathrm{s}(\mathrm{p}>0.05)$. CONCLUSION: The findings indicate that both training conditions resulted in similar dynamic strength gains suggesting that low-intensity BFR training is as effective as high-intensity training in neuromuscular adaptation following short-term resistance training. The results also suggest that non-BFR condition placed an increased demand on the cardiovascular system, but subjects experienced higher perceived exertion during BFR. 